\title{
AVALIAÇÃO DE GENÓTIPOS DE BANANEIRA NA REGIÃO DO BAIXO SÃO FRANCISCO, SERGIPE ${ }^{1}$
}

\author{
ANADA SILVALÉDO², JOSUÉ FRANCISCO DA SILVAJUNIOR ${ }^{3}$, \\ CARLOS ALBERTO DA SILVALÉDO ${ }^{4}$, SEBASTIÃO DE OLIVEIRAE SILVA ${ }^{4}$
}

RESUMO- Este trabalho teve como objetivo avaliar o desempenho de 20 genótipos de bananeira nas condições edafoclimáticas da região do Baixo São Francisco-SE. O experimento foi instalado em área experimental da Embrapa Tabuleiros Costeiros, no município de Propriá- SE. O delineamento foi em blocos ao acaso, com quatro repetições e três touceiras úteis por parcela. Foram avaliados caracteres de crescimento e produção no primeiro e segundo ciclos. Os híbridos PV42-53, PV42-68, PV42-85 e FHIA-02, devido ao bom desempenho com relação ao peso do cacho e peso médio do fruto, podem ser recomendados para cultivo no Baixo São Francisco. O híbrido FHIA-18 apresentou excelentes características agronômicas, sendo uma alternativa a cultivar Prata-Anã. Os híbridos Ambrósia e Bucaneiro podem ser indicados por apresentarem cachos grandes e sabor semelhante às bananas do subgrupo Cavendish. $\mathrm{O}$ híbrido YB42-07 tem potencial para ser lançado como cultivar em áreas de cultivo de banana Maçã.

Termos para indexação: Musa sp., variedades, híbridos.

\section{EVALUATION OF BANANA GENOTYPES IN THE LOW SÃO FRANCISCO RIVER BASIN, SERGIPE, BRAZIL}

ABSTRACT- The main objective of this work was evaluated the performance of twenty genotypes of Musa sp., in the environmental conditions of the Low São Francisco River Basin, Propriá, SE, and Brazil. The experimental design was a randomized complete block with four replications and three useful plants per parcel. rowth and yield characteristics were analyzed during the first and second production cycles. Hybrids PV42-53, PV42-68, PV42-85 and FHIA-02 because of the bigger production and greater weight of the fruit, should be recommended for culture in the region. Hybrid FHIA-18 presented excellent agronomic characteristics, being an alternative to 'Prata Anã'. The hybrids Ambrosia and Buccaneer can be an alternative for Cavendish bananas, by showing great bunches and similar flavor. Hybrid YB42-07 could be an option for areas of 'Silk' banana culture in the region of the Low São Francisco River, having potential to be set up as cultivar.

Index terms: Musa sp., cultivars, hybrids.

\section{INTRODUÇÃO}

A cultura da banana assume importância econômica e social em todo o mundo, sendo cultivada em mais de 80 países tropicais. O Brasil é o terceiro produtor mundial, sendo a banana a segunda fruta mais consumida no País, com uma produção aproximada de 6,8 milhões de toneladas, em uma área cultivada de 500 mil hectares (FAO, 2007).

A região Nordeste é responsável pela maior produção, com 2,7 milhões de toneladas, ocupando uma área de 210.374 ha, correspondendo a $38,6 \%$ da produção total do País, seguida pelo Sudeste, com $29,8 \%$ da produção (IBGE, 2008). A bananicultura possui uma grande importância socioeconômica no Nordeste, sendo geralmente explorada por pequenos agricultores, predominando a mão-de-obra familiar. Constitui parte integrante da alimentação de populações de baixa renda, não só pelo seu alto valor nutritivo, mas também pelo seu baixo custo, tendo papel fundamental na fixação da mão-de-obra rural (Dantas et al., 1999).
Dentre as áreas de produção de banana em Sergipe, destacam-se as microrregiões do Agreste de Itabaiana, Cotinguiba, Baixo Cotinguiba, Japaratuba, Estância e Boquim, com $78,7 \%$ da área colhida no Estado, sendo as microrregiões do Baixo São Francisco responsáveis por 43,6\% da produção (IBGE, 2008).

Os problemas fitossanitários constituem a maior ameaça para a cultura, tendo em vista a utilização generalizada das cultivares Prata e Maçã, suscetíveis a diversas doenças, como a Sigatoka-amarela, causada por Mycosphaerella musicola Leach, e mal-do-Panamá, cujo agente causal é o Fusarium oxysporum $\mathrm{f}$. sp. cubense (E.F. Smith) Snyder \& Hansen. Além dessas enfermidades, a Sigatoka-negra, causada por Mycosphaerella fijiensis Morelet, principal problema da bananicultura mundial, constitui fator limitante para a expansão da cultura na região Nordeste. Uma estratégia para a solução deste problema é o desenvolvimento de novas cultivares mais produtivas e resistentes, mediante o melhoramento genético, cuja etapa final do processo consiste na avaliação dos genótipos em diferentes regiões produtoras (Silva et al., 2002; Silva Junior et al., 2002;

'(Trabalho 169-07). Recebido em: 12-07-2007. Aceito para publicação em: 22-04-2008.

${ }^{2}$ Eng. Agrônoma, D.Sc. Pesquisadora da Embrapa Tabuleiros Costeiros, Caixa Postal 44, CEP 49025-040, Aracaju - SE. E-mail: analedo@cpatc.embrapa.br; ${ }^{3}$ Eng. Agrônomo, M.Sc., Pesquisador da Embrapa Tabuleiros Costeiros. E-mail: josue@cpatc.embrapa.br;

${ }^{4}$ Eng. Agrônomo, D.Sc., pesquisador da Embrapa Mandioca e Fruticultura Tropical, Caixa Postal 007, CEP 44380-000-Cruz das Almas-BA. E-mail: ledo@cnpmf.embrapa.br; ssilva@cnpmf.embrapa.br. 
Donato et al., 2003; Pereira et al., 2003; Lima et al., 2005; Rodrigues et al., 2006; Silva et al., 2006).

O objetivo do trabalho foi avaliar genótipos de bananeira nas condições edafoclimáticas da região do Baixo São Francisco visando à seleção de materiais com características agronômicas e comerciais superiores.

\section{MATERIAL E MÉTODOS}

O trabalho foi instalado em outubro de 2004, na área experimental da Embrapa Tabuleiros Costeiros, no perímetro irrigado Cotinguiba-Pindoba, no município de Propriá, no Baixo São Francisco-SE, em Neossolo Flúvico de textura argilosa e de fertilidade média. O clima da região é semi-úmido, com chuvas predominantes de inverno e outono, apresentando médias anuais de $1.161 \mathrm{~mm}$, sendo que $74 \%$ são distribuídas de abril a setembro. A temperatura média do ar é de $25^{\circ} \mathrm{C}$, e a umidade relativa, de $77 \%$.

O delineamento experimental foi o de blocos casualizados, com 20 tratamentos (cultivares e híbridos) e quatro repetições, sendo cada parcela formada por cinco touceiras, e a parcela útil, por três touceiras. Os tratamentos foram compostos pelos seguintes genótipos: híbridos tetraplóides de 'Gros Michel' (Bucaneiro e Ambrosia); 'Yangambi n' 2' (YB42-07 e YB42-21); 'Prata São Tomé'(ST42-08); 'Prata-Anã'(PA42-44, PA42-28, PA4219, PA42-38D e FHIA-18); 'Pacovan' (PV42-53, PV42-85 e PV4268), Cavendish (FHIA-02); e as cultivares Pacovan e Prata-Anã (subgrupo Prata), Grande Naine (subgrupo Cavendish), Maçã, Caipira (subgrupo Ibota) e Thap Maeo. As mudas micropropagadas, oriundas da Embrapa Mandioca e Fruticultura Tropical, foram plantadas no espaçamento $3,00 \mathrm{~m} \times 2,00 \mathrm{~m}$, em sistema de irrigação por aspersão convencional, atendendo à demanda hídrica da cultura. Foram realizados os tratos culturais de acordo com as recomendações técnicas (Alves \& Oliveira, 1999). No segundo ciclo de produção, em virtude da perda de três parcelas, foram avaliadas 17 cultivares e híbridos.

Por ocasião do florescimento, foram avaliadas as seguintes características: altura da planta do nível do solo à inserção da inflorescência, perímetro do pseudocaule a $30 \mathrm{~cm}$ do solo, número de folhas vivas, número de dias do plantio à emissão da inflorescência no primeiro ciclo. Na colheita: o número de folhas vivas, peso do cacho, número de pencas/cacho, número de frutos/cacho, número de frutos/penca, peso das pencas, peso médio da penca, peso médio dos frutos, número de dias do plantio à colheita do primeiro ciclo e número de dias do plantio à colheita do segundo ciclo. Foi realizada a análise de variância, e as médias, agrupadas pelo teste de Scott-Knot, a 5\% de significância.

\section{RESULTADOS E DISCUSSÃO}

A análise de variância foi significativa para todas as características avaliadas no primeiro e segundo ciclos, resultado já esperado considerando que, dentre os materiais avaliados, encontram-se genótipos oriundos de diferentes grupos e subgrupos (Tabelas 1; 2 e 3 ). Na bananeira, a variabilidade genética importante localiza-se entre as diversas formas selvagens da espécie Musa acuminata e nas cultivares do grupo AA, que apresentam grande diversidade morfológica com variações no porte, vigor de perfilhamento, número de pencas por cacho e no tamanho dos frutos (Sherpherd et al., citados por Dantas et al., 1999).

No primeiro ciclo, verificou-se a formação de quatro grupos quanto à altura da planta. No primeiro, porte alto, classificaram-se PV42-85, PV42-53, PV42-68 e a Maçã, com médias que variaram de $3,42 \mathrm{~m}$ a $3,75 \mathrm{~m}$ (Tabela 1). Os genótipos PrataAnã, Grande Naine, FHIA-02, PA42-28 e PA42-44 foram reunidos no grupamento que apresentou o menor porte, com médias entre $2,08 \mathrm{~m}$ e 2,42 m. No segundo ciclo, foi constatada a formação de três grupos, sendo os híbridos de PV42-68, PV42-85, PV42-53, ST 42-08 e cultivar Pacovan de porte alto, e os genótipos PA4228, PA42-44, PA42-19, FHIA-18, FHIA-02, Prata-Anã e Bucaneiro, de porte baixo (Tabela 2). Nesse ciclo, a altura das plantas variou de 3,45 a 4,99 m, observando um aumento médio de $65,86 \%$ a $106,20 \%$ na altura dos genótipos. O aumento e a estabilidade dessa característica, no segundo ciclo, também já foram observados por outros autores (Silva et al., 2002; Silva et al., 2003; Donato et al., 2003; Lima et al., 2005). O perímetro do pseudocaule também apresentou aumento dos valores no segundo ciclo (Tabelas 1 e 2), destacando-se o híbrido tetraplóide Ambrosia, seguido dos híbridos PV42-85, PV42-53, PV42-68, PA42-19, PA42-38D, ST42-08 e das cultivares Prata-Anã e Thap Maeo. Ambos os caracteres são importantes para a seleção de genótipos promissores por estarem relacionados com a densidade de plantio e a capacidade de sustentação do cacho (Silva Junior et al., 2002; Silva et al., 2002).

O número de folhas vivas no florescimento variou de 11,7 a 19,5 e de 10,6 a 12,5 no primeiro e segundo ciclos, respectivamente. A diminuição no número de folhas ativas no segundo ciclo pode ser devida à alta incidência de Sigatokaamarela na região, fato também observado por Vieira Neto (2001). $\mathrm{Na}$ colheita, registrou-se uma variação de 6,7 a 10,6 e de 7,1 a 10,0 folhas/planta no primeiro e segundo ciclos, respectivamente. Observou-se uma redução drástica do número de folhas ativas na colheita, durante os dois ciclos, sendo que, no segundo ciclo, apenas a cultivar Thap Maeo apresentou o mínimo de 10 folhas/ planta (Tabela 2). $\mathrm{O}$ enchimento dos frutos está diretamente correlacionado ao número de folhas vivas na colheita (Lima et al., 2005). Entretanto, os genótipos que obtiveram maior número de folhas vivas na colheita, não apresentaram maior peso de cacho (Tabelas 1 e 2). Resultados semelhantes foram observados por Lima et al. (2005) na região do Recôncavo Baiano, com exceção do híbrido Bucaneiro. Este fato pode ser devido às características genéticas das cultivares e/ou híbridos, ou seja, alguns genótipos podem apresentar bom desenvolvimento dos frutos com menor número de folhas ativas após o florescimento.

No primeiro ciclo, o peso de cacho variou de 13,67 a 29,98 $\mathrm{kg}$, respectivamente, para os genótipos PA42-44 e Bucaneiro, e o peso médio do fruto, de 93,40 na cultivar Thap Maeo, a 209,9 g para o híbrido PV42-53, enquanto o número de frutos variou de 71,7 para ST42-08 a 201,5 para a cultivar Thap Maeo (Tabela 1). O peso de cacho depende do número de frutos por cacho e do peso médio dos mesmos. Dessa forma, genótipos com maior 
número de frutos/cacho e de maior peso médio apresentarão maior produção por cacho. Entretanto, a associação dessas duas características não foi observada para algumas cultivares e híbridos no presente estudo, corroborando os resultados obtidos por Lima et al. (2005). O número de frutos produzidos é fundamental na determinação do peso do cacho, podendo ser o fator que melhor explica essa característica (Lima et al., 2005). Estudos sobre a associação entre esses dois caracteres conduzidos por Flores (2000) reforçam a importância da avaliação do caractere número de frutos para o melhoramento genético dessa frutífera.

Os maiores pesos de cacho no primeiro ciclo foram obtidos pelos genótipos Bucaneiro, Ambrosia e Grande Naine, com 29,98; 25,48 e 24,19 kg, respectivamente (Tabela 1). Esses genótipos pertencem aos subgrupos Gros Michel e Cavendish, que produzem os maiores cachos entre as bananeiras comestíveis (Silva et al., 1999).

No segundo ciclo, o peso de cacho variou de 13,29 a $23,00 \mathrm{~kg}$, para os genótipos ST42-08 e FHIA-02, respectivamente. Em relação ao peso médio de fruto, ocorreu variação de $86,7 \mathrm{~g}$ para a cultivar Thap Maeo, a 179,2 g para o híbrido PV42-85, enquanto o número de frutos variou de 78,0 para ST42-08, a 223,7 para Thap Maeo (Tabela 2). Frutos grandes são uma das exigências do mercado nordestino, que tem preferência pela cultivar Pacovan; nesse sentido, os híbridos PV42-53, PV42-85 e PV42-68 apresentaram bom desempenho com relação ao peso do cacho e peso médio do fruto, nos dois ciclos de produção.

Os genótipos que alcançaram as maiores produções, no segundo ciclo, foram FHIA-02, FHIA-18, Thap Maeo e Ambrósia, com 23,$00 ; 20,15 ; 20,85$ e 20,28 kg, respectivamente. Os valores médios de peso do cacho, no primeiro ciclo, dos genótipos Ambrosia, Bucaneiro, Pacovan e ST42-08 foram superiores aos obtidos por Lima et al. (2005) no Recôncavo Baiano. Entretanto, esse comportamento não foi detectado no segundo ciclo de produção. Provavelmente, a redução do número de folhas vivas por ocasião da colheita do segundo ciclo pode ter influenciado na produção desses genótipos.

As cultivares Pacovan, Prata-Anã, Thap Maeo, Caipira e o híbrido FHIA-18 apresentaram, em média, maior peso do cacho nos dois ciclos quando comparadas com os resultados obtidos por Silva et al. (2002), em Cruz das Almas-BA, em Latossolo Amarelo sob microaspersão, e por Silva Junior et al. (2002), nas condições de clima e solo da Zona da Mata, em Pernambuco. A diferença entre cultivares e entre regiões pode ocorrer devido ao tipo e idade da muda, bem como pelas condições ambientais, como altitude, luminosidade, temperatura e umidade (Simão, 1998). Considerando que diferentes ambientes influenciam no desempenho dos genótipos de banana, na manifestação dos caracteres, o melhor comportamento desses genótipos reforça a sua boa adaptação às condições edafoclimáticas da região do Baixo São Francisco.

No primeiro e segundo ciclos, os híbridos de Pacovan foram mais tardios que sua genitora. Esse comportamento também foi observado para os híbridos de Prata-Anã durante o primeiro ciclo; entretanto, no segundo ciclo, os híbridos PA42-44, PA4219, PA42-38D e FHIA-18 apresentaram precocidade quando comparados com a sua progenitora (Tabela 3). Esses resultados divergem daqueles obtidos por Silva et al. (2002), que observaram precocidade dos híbridos em relação aos seus progenitores, em ambos os ciclos. O ciclo é um caráter de relevância no melhoramento genético da bananeira por refletir a precocidade da planta (Silva et al., 2002). A precocidade dita retorno econômico mais rápido ao produtor, enquanto o menor tempo de permanência na planta reduz o tempo de exposição do cacho a agentes causadores de danos (Rodrigues et al., 2006). As condições climáticas e principalmente os genótipos têm influência significativa no ciclo (Alves et al., 1999; Silva et al., 1999), além da densidade de plantas (Scarpare Filho \& Kluge, 2001).

TABELA 1 - Médias dos caracteres altura da planta (ALT), perímetro do pseudocaule a $30 \mathrm{~cm}$ do solo (PER), número de folhas vivas na floração (NFVF), número de folhas vivas na colheita (NFVC), peso do cacho (PC), peso das pencas (PP), número de pencas por cacho (NPC), número de frutos por cacho (NFC), número de frutos por penca (NFP), peso médio da penca (PMP) e peso médio do fruto (PMF) de genótipos de bananeira, no primeiro ciclo de produção. Propriá-SE, 2005-2006.

\begin{tabular}{|c|c|c|c|c|c|c|c|c|c|c|c|c|c|c|c|c|c|c|c|c|c|c|}
\hline Genótipos & ALT & & PER ( & m) & NFV & & NFVC & & PC (ks & & PP (kg & & NPC & & NFC & & NF & & PMP (k & & PMF (g & \\
\hline PV42-85 & 3,75 & $\mathrm{a}$ & 74,50 & $\mathrm{~b}$ & 15,8 & $\mathrm{c}$ & 7,3 & $\mathrm{c}$ & 18,53 & $\mathrm{c}$ & 17,17 & $\mathrm{c}$ & 6,3 & & 85,1 & $\mathrm{e}$ & 13,5 & $\mathrm{c}$ & 2,76 & & 203,8 & $\mathrm{a}$ \\
\hline PV42-53 & 3,67 & a & 77,17 & b & 16,0 & $\mathrm{c}$ & 7,7 & $\mathrm{~b}$ & 19,66 & $\mathrm{c}$ & 18,24 & $\mathrm{c}$ & 6,3 & $\mathrm{e}$ & 87,6 & $\mathrm{e}$ & 14,0 & $\mathrm{c}$ & 2,92 & b & 209,9 & $\mathrm{a}$ \\
\hline PV42-68 & 3,50 & a & 68,25 & $\mathrm{c}$ & 15,0 & $\mathrm{c}$ & 7,7 & b & 18,13 & $\mathrm{c}$ & 16,60 & $\mathrm{c}$ & 6,2 & $\mathrm{e}$ & 83,8 & $\mathrm{e}$ & 13,6 & $\mathrm{c}$ & 2,70 & b & 197,8 & $\mathrm{a}$ \\
\hline Pacovan & 3,08 & b & 64,67 & d & 18,7 & $\mathrm{a}$ & 6,9 & $\mathrm{c}$ & 14,33 & d & 12,89 & d & 6,4 & $\mathrm{e}$ & 78,8 & $\mathrm{e}$ & 12,3 & d & 2,02 & $\mathrm{c}$ & 164,4 & $\mathrm{~b}$ \\
\hline PA $42-44$ & 2,33 & d & 66,08 & d & 17,3 & b & 8,3 & $\mathrm{~b}$ & 13,67 & d & 12,49 & d & 6,8 & d & 84,0 & $\mathrm{e}$ & 12,3 & d & 1,83 & d & 150,3 & $\mathrm{c}$ \\
\hline PA42-19 & 2,67 & $\mathrm{c}$ & 69,08 & $\mathrm{c}$ & 16,5 & $\mathrm{c}$ & 6,2 & $\mathrm{c}$ & 13,99 & d & 12,86 & d & 6,8 & d & 90,7 & $\mathrm{e}$ & 13,4 & $\mathrm{c}$ & 1,90 & d & 142,8 & $\mathrm{c}$ \\
\hline PA42-28 & 2,42 & d & 66,33 & d & 17,5 & $\mathrm{~b}$ & 8,2 & $\mathrm{~b}$ & 13,73 & d & 12,49 & d & 6,8 & d & 81,7 & $\mathrm{e}$ & 12,0 & d & 1,85 & d & 155,8 & $\mathrm{c}$ \\
\hline PA42-38D & 2,75 & $\mathrm{c}$ & 69,33 & $\mathrm{c}$ & 16,1 & $\mathrm{c}$ & 7,2 & $\mathrm{c}$ & 13,78 & d & 12,45 & d & 7,1 & d & 100,3 & $\mathrm{~d}$ & 14,2 & $\mathrm{c}$ & 1,77 & d & 125,4 & $\mathrm{c}$ \\
\hline FHIA-18 & 2,50 & $\mathrm{c}$ & 66,67 & d & 16,0 & $\mathrm{c}$ & 10,1 & a & 18,42 & $\mathrm{c}$ & 16,73 & $\mathrm{c}$ & 8,8 & $\mathrm{~b}$ & 126,7 & c & 14,5 & $\mathrm{c}$ & 1,91 & d & 132,9 & $\mathrm{c}$ \\
\hline ST42-08 & 3,25 & b & 63,92 & d & 14,6 & d & 8,2 & $\mathrm{~b}$ & 13,71 & d & 12,77 & d & 6,0 & $\mathrm{e}$ & 71,8 & $\mathrm{e}$ & 12,0 & d & 2,16 & c & 178,4 & $\mathrm{~b}$ \\
\hline Prata-Anã & 2,08 & d & 70,17 & $\mathrm{c}$ & 19,8 & $\mathrm{a}$ & 8,6 & $\mathrm{~b}$ & 14,40 & d & 1,47 & $\mathrm{~b}$ & 12,9 & & 108,3 & d & 13,3 & $\mathrm{c}$ & 1,56 & d & 116,3 & d \\
\hline YB42-07 & 3,08 & b & 78,58 & b & 15,6 & $\mathrm{c}$ & 10,6 & & 17,57 & $\mathrm{c}$ & 16,13 & $\mathrm{c}$ & 7,8 & $\mathrm{c}$ & 125,3 & $\mathrm{c}$ & 16,1 & b & 2,08 & $\mathrm{c}$ & 132,4 & $\mathrm{c}$ \\
\hline YB42-21 & 3,25 & b & 85,83 & a & 13,3 & $\mathrm{e}$ & 8,51 & $\mathrm{~b}$ & 17,99 & $\mathrm{c}$ & 16,77 & $\mathrm{c}$ & 6,3 & $\mathrm{e}$ & 92,3 & $\mathrm{e}$ & 14,8 & $\mathrm{c}$ & 2,67 & b & 187,7 & a \\
\hline FHIA-02 & 2,25 & d & 65,25 & d & 15,5 & $\mathrm{c}$ & 8,4 & $\mathrm{~b}$ & 18,10 & $\mathrm{c}$ & 16,51 & $\mathrm{c}$ & 9,2 & $\mathrm{~b}$ & 128,0 & $\mathrm{c}$ & 14,0 & $\mathrm{c}$ & 1,80 & d & 129,2 & $\mathrm{c}$ \\
\hline Grande Nine & 2,17 & d & 63,42 & d & 15,3 & $\mathrm{c}$ & 8,1 & $\mathrm{~b}$ & 24,19 & $\mathrm{~b}$ & 22,25 & $\mathrm{~b}$ & 8,2 & $\mathrm{c}$ & 119,2 & $\mathrm{c}$ & 14,4 & $\mathrm{c}$ & 2,72 & b & 189,4 & $\mathrm{a}$ \\
\hline Thap Maeo & 3,08 & $\mathrm{~b}$ & 72,33 & $\mathrm{c}$ & 16,4 & $\mathrm{c}$ & 9,8 & $\mathrm{a}$ & 20,12 & $\mathrm{c}$ & 18,61 & $\mathrm{c}$ & 11,7 & a & 201,5 & $\mathrm{a}$ & 17,1 & b & 1,58 & d & 93,4 & d \\
\hline Caipira & 3,25 & b & 71,17 & $\mathrm{c}$ & 12,6 & $\mathrm{e}$ & 6,6 & $\mathrm{c}$ & 17,84 & $\mathrm{c}$ & 16,53 & $\mathrm{c}$ & 7,5 & d & 143,5 & $\mathrm{~b}$ & 19,1 & a & 2,20 & $\mathrm{c}$ & 114,6 & d \\
\hline Maçã & 3,42 & a & 78,58 & b & 15,9 & $\mathrm{c}$ & 7,0 & $\mathrm{c}$ & 18,00 & $\mathrm{c}$ & 16,45 & $\mathrm{c}$ & 7,8 & $\mathrm{c}$ & 125,3 & $\mathrm{c}$ & 16,0 & b & 2,09 & $\mathrm{c}$ & 130,8 & $\mathrm{c}$ \\
\hline Ambrosia & 3,00 & b & 75,25 & b & 12,9 & $\mathrm{e}$ & 7,3 & $\mathrm{c}$ & 25,48 & $\mathrm{~b}$ & 23,28 & $\mathrm{~b}$ & 8,3 & $\mathrm{c}$ & 138,3 & b & 16,5 & b & 2,78 & b & 167,7 & $\mathrm{~b}$ \\
\hline Bucaneiro & 3,00 & $\mathrm{~b}$ & 75,33 & $\mathrm{~b}$ & 11,7 & $\mathrm{e}$ & $8,4 \quad 1$ & & 29,98 & $\mathrm{a}$ & 27,83 & $\mathrm{a}$ & 8,4 & & 149,2 & $\mathrm{~b}$ & 17,8 & $\mathrm{a}$ & 3,34 & $\mathrm{a}$ & 187,9 & \\
\hline CV $(\%)$ & 14,7 & & 7,27 & & 12,2 & & 22,77 & & 18,08 & & 16,60 & & 7,53 & & 22,7 & & 18,2 & & 21,17 & & 22,71 & \\
\hline
\end{tabular}

Médias seguidas pela mesma letra na coluna pertencem ao mesmo grupo, pelo teste de Scott-Knot, a $5 \%$ de probabilidade. 
TABELA 2 - Médias dos caracteres altura da planta (ALT), perímetro do pseudocaule a $30 \mathrm{~cm}$ do solo (PER), número de folhas vivas na floração (NFVF), número de folhas vivas na colheita (NFVC), peso do cacho (PC), peso das pencas (PP), número de pencas por cacho (NPC), número de frutos por cacho (NFC), número de frutos por penca (NFP), peso médio da penca (PMP) e peso médio do fruto (PMF) de genótipos de bananeira, no segundo ciclo de produção. Propriá-SE, 2005-2006.

\begin{tabular}{|c|c|c|c|c|c|c|c|c|c|c|c|c|c|c|c|c|c|c|}
\hline \multirow{2}{*}{$\begin{array}{l}\text { Genótipos } \\
\text { PV42-85 }\end{array}$} & ALT (m) & PER $(\mathrm{cm})$ & \multicolumn{2}{|c|}{ NFVF } & \multicolumn{2}{|c|}{ NFVC } & \multicolumn{2}{|c|}{$\mathrm{PC}(\mathrm{kg})$} & \multicolumn{2}{|c|}{$\mathrm{PP}(\mathrm{kg})$} & \multicolumn{2}{|c|}{ NPC } & NFC & \multicolumn{2}{|c|}{ NFP } & \multicolumn{2}{|c|}{ PMP (kg) } & PMF (g) \\
\hline & $\begin{array}{ll}4,99 \quad \mathrm{a} \\
\end{array}$ & $93,50 \mathrm{~b}$ & 11,8 & $\mathrm{a}$ & 8,2 & & 18,18 & $\mathrm{~b}$ & 16,82 & $\mathrm{~b}$ & 6,8 & $\mathrm{e}$ & $94,0 \quad \mathrm{e}$ & 13,8 & $\mathrm{c}$ & 2,46 & & 179,2 a \\
\hline PV42-53 & $4,71 \quad \mathrm{a}$ & 88,42 b & 11,7 & $\mathrm{a}$ & 8,5 & $\mathrm{~b}$ & 19,65 & b & 18,20 & b & 7,3 & $\mathrm{~d}$ & $103,5 \mathrm{e}$ & 14,3 & $\mathrm{c}$ & 2,52 & $\mathrm{a}$ & $175,0 \quad \mathrm{a}$ \\
\hline PV42-68 & 4,94 a & $89,00 \quad \mathrm{~b}$ & 12,5 & $\mathrm{a}$ & 8,5 & $\mathrm{~b}$ & 16,00 & $\mathrm{c}$ & 14,80 & $\mathrm{c}$ & 6,4 & $\mathrm{e}$ & $85,8 \mathrm{e}$ & 13,4 & d & 2,33 & $\mathrm{a}$ & 175,8 a \\
\hline Pacovan & 4,80 a & $82,92 \quad \mathrm{c}$ & 11,8 & a & 7,3 & $\mathrm{c}$ & 15,12 & $\mathrm{c}$ & 13,86 & $\mathrm{c}$ & 7,8 & $\mathrm{c}$ & $100,9 \mathrm{e}$ & 13,0 & d & 2,02 & $\mathrm{c}$ & $136,7 \quad b$ \\
\hline PA42-44 & 3,52 & $83,33 \mathrm{c}$ & 11,7 & $\mathrm{a}$ & 8,5 & $\mathrm{~b}$ & 14,72 & $\mathrm{c}$ & 13,48 & $\mathrm{c}$ & 7,2 & d & $92,6 \mathrm{e}$ & 12,9 & d & 1,89 & $\mathrm{~b}$ & $146,7 \mathrm{~b}$ \\
\hline PA42-19 & $3,66 \mathrm{c}$ & 89,42 b & 11,6 & $\mathrm{a}$ & 8,4 & $\mathrm{~b}$ & 16,38 & $\mathrm{c}$ & 15,13 & $\mathrm{c}$ & 7,5 & $\mathrm{~d}$ & $104,5 \mathrm{e}$ & 13,9 & $\mathrm{c}$ & 2,05 & $\mathrm{~b}$ & $150,0 \mathrm{~b}$ \\
\hline PA42-28 & $3,49 \mathrm{c}$ & $81,33 \mathrm{c}$ & 12,3 & $\mathrm{a}$ & 9,0 & $\mathrm{~b}$ & 14,51 & $\mathrm{c}$ & 12,34 & $\mathrm{c}$ & 7,3 & $\mathrm{~d}$ & $93,3 \mathrm{e}$ & 12,8 & d & 1,88 & $\mathrm{c}$ & $147,5 \mathrm{~b}$ \\
\hline PA42-38D & $3,78 \mathrm{~b}$ & $92,00 \quad b$ & 12,3 & $\mathrm{a}$ & 8,4 & $\mathrm{~b}$ & 18,28 & $\mathrm{~b}$ & 16,72 & $\mathrm{~b}$ & 8,3 & $\mathrm{c}$ & $123,8 \mathrm{~d}$ & 15,0 & $\mathrm{c}$ & 2,04 & $\mathrm{~b}$ & $137,5 \mathrm{~b}$ \\
\hline FHIA-18 & $3,46 \mathrm{c}$ & $81,00 \quad \mathrm{c}$ & 10,6 & $\mathrm{a}$ & 7,6 & $\mathrm{c}$ & 20,15 & $\mathrm{a}$ & 18,72 & $\mathrm{~b}$ & 9,3 & b & $129,8 \mathrm{~d}$ & 13,9 & $\mathrm{c}$ & 2,02 & b & $144,2 \mathrm{~b}$ \\
\hline ST42-08 & 4,93 a & 88,25 b & 11,9 & $\mathrm{a}$ & 7,7 & $\mathrm{c}$ & 13,29 & $\mathrm{c}$ & 12,31 & $\mathrm{c}$ & 6,3 & $\mathrm{e}$ & $78,0 \mathrm{e}$ & 12,3 & d & 1,95 & $\mathrm{~b}$ & 158,3 b \\
\hline Prata-Anã & $3,23 \mathrm{c}$ & $87,50 \quad b$ & 11,9 & $\mathrm{a}$ & 8,2 & $\mathrm{~b}$ & 15,82 & $\mathrm{c}$ & 14,78 & $\mathrm{c}$ & 8,6 & c & $117,7 \mathrm{~d}$ & 13,7 & $\mathrm{c}$ & 1,72 & d & $116,3 \mathrm{~d}$ \\
\hline YB42-07 & $4,08 \quad \mathrm{~b}$ & $84,33 \mathrm{c}$ & 11,9 & $\mathrm{a}$ & 8,8 & $\mathrm{~b}$ & 13,59 & $\mathrm{c}$ & 12,17 & $\mathrm{c}$ & 8,3 & $\mathrm{a}$ & $123,4 \mathrm{~b}$ & 14,8 & $\mathrm{c}$ & 1,47 & $\mathrm{e}$ & $100,3 \mathrm{~d}$ \\
\hline FHIA-02 & $3,45 \mathrm{c}$ & $81,33 \mathrm{c}$ & 11,6 & $\mathrm{a}$ & 7,7 & $\mathrm{c}$ & 23,00 & $\mathrm{a}$ & 21,52 & $\mathrm{a}$ & 9,8 & b & $139,9 \mathrm{~d}$ & 14,4 & $\mathrm{c}$ & 2,22 & $\mathrm{a}$ & $155,0 \quad \mathrm{~b}$ \\
\hline Thap Maeo & $4,16 \mathrm{~b}$ & 88,92 b & 12,2 & $\mathrm{a}$ & 10,0 & $\mathrm{a}$ & 20,28 & $\mathrm{a}$ & 18,68 & $\mathrm{~b}$ & 13,6 & a & $223,7 \mathrm{a}$ & 16,3 & b & 1,38 & $\mathrm{e}$ & $86,7 \mathrm{~d}$ \\
\hline Caipira & $4,03 \mathrm{~b}$ & $77,17 \quad \mathrm{c}$ & 11,3 & $\mathrm{a}$ & 8,4 & $\mathrm{~b}$ & 18,15 & $\mathrm{~b}$ & 16,67 & $\mathrm{~b}$ & 9,8 & $\mathrm{~b}$ & $188,7 \mathrm{~b}$ & 19,2 & $\mathrm{a}$ & 1,70 & d & $89,2 \mathrm{~d}$ \\
\hline Ambrosia & $4,01 \quad \mathrm{~b}$ & $100,42 \mathrm{a}$ & 11,4 & $\mathrm{a}$ & 8,0 & $\mathrm{c}$ & 20,85 & $\mathrm{a}$ & 18,99 & $\mathrm{~b}$ & 9,9 & $\mathrm{~b}$ & $159,2 \mathrm{c}$ & 16,0 & $\mathrm{~b}$ & 2,78 & $\mathrm{~b}$ & $120,1 \mathrm{c}$ \\
\hline Bucaneiro & $3,45 \mathrm{c}$ & $90,83 \mathrm{~b}$ & 11,0 & $\mathrm{a}$ & 7,1 & $\mathrm{c}$ & 18,72 & $\mathrm{~b}$ & 17,31 & $\mathrm{~b}$ & 8,9 & $\mathrm{~b}$ & $152,1 \mathrm{c}$ & 17,0 & $\mathrm{~b}$ & 1,95 & $\mathrm{~b}$ & $125,8 \mathrm{c}$ \\
\hline CV (\%) & 8,36 & 8,35 & 10,3 & & 14,0 & & 20,3 & & 20,88 & & 12,5 & & 19,35 & 10,4 & & 18,8 & & 18,68 \\
\hline
\end{tabular}

Médias seguidas pela mesma letra na coluna pertencem ao mesmo grupo, pelo teste de Scott-Knot, a 5\% de probabilidade.

TABELA 3- Médias de caracteres referentes ao número de dias do plantio ao florescimento no primeiro ciclo (DPF1), número de dias do plantio à colheita no primeiro ciclo (DPC1), número de dias do plantio ao florescimento no segundo ciclo (DPF2) e número de dias do plantio à colheita no segundo ciclo (DPC2) de genótipos de bananeira. PropriáSE, 2005-2006.

\begin{tabular}{ccccc}
\hline Genótipos & DPF1 & DPC1 & DPF2 & DPC2 \\
\hline PV42-85 & $261,1 \mathrm{~b}$ & $393,6 \mathrm{~b}$ & $534,8 \mathrm{a}$ & $622,8 \mathrm{a}$ \\
PV42-53 & $275,8 \mathrm{~b}$ & $411,5 \mathrm{a}$ & $585,8 \mathrm{a}$ & $651,8 \mathrm{a}$ \\
PV42-68 & $249,7 \mathrm{c}$ & $370,3 \mathrm{~b}$ & $505,2 \mathrm{~b}$ & $579,6 \mathrm{~b}$ \\
Pacovan & $240,3 \mathrm{c}$ & $359,5 \mathrm{~b}$ & $422,5 \mathrm{c}$ & $552,1 \mathrm{~b}$ \\
PA42-44 & $223,3 \mathrm{~d}$ & $412,8 \mathrm{a}$ & $457,1 \mathrm{~b}$ & $568,5 \mathrm{~b}$ \\
PA42-19 & $232,3 \mathrm{c}$ & $387,8 \mathrm{~b}$ & $470,3 \mathrm{~b}$ & $578,0 \mathrm{~b}$ \\
PA42-28 & $217,8 \mathrm{~d}$ & $372,8 \mathrm{~b}$ & $492,4 \mathrm{~b}$ & $570,4 \mathrm{~b}$ \\
PA42-38D & $220,2 \mathrm{~d}$ & $425,5 \mathrm{a}$ & $434,0 \mathrm{c}$ & $536,9 \mathrm{~b}$ \\
FHIA-18 & $229,6 \mathrm{~d}$ & $358,6 \mathrm{~b}$ & $478,0 \mathrm{~b}$ & $589,1 \mathrm{~b}$ \\
ST42-08 & $253,3 \mathrm{c}$ & $380,9 \mathrm{~b}$ & $505,9 \mathrm{~b}$ & $623,0 \mathrm{a}$ \\
Prata-Anã & $207,8 \mathrm{~d}$ & $360,5 \mathrm{~b}$ & $479,4 \mathrm{~b}$ & $592,8 \mathrm{~b}$ \\
YB42-07 & $286,2 \mathrm{~b}$ & $387,3 \mathrm{~b}$ & $505,8 \mathrm{~b}$ & $599,8 \mathrm{~b}$ \\
YB42-21 & $317,0 \mathrm{a}$ & $414,9 \mathrm{a}$ & - & - \\
FHIA-02 & $218,5 \mathrm{~d}$ & $372,5 \mathrm{~b}$ & $389,7 \mathrm{c}$ & $481,8 \mathrm{c}$ \\
Grande Nine & $237,7 \mathrm{c}$ & $376,8 \mathrm{~b}$ & - & - \\
Thap Maeo & $273,7 \mathrm{~b}$ & $377,7 \mathrm{~b}$ & $608,0 \mathrm{a}$ & - \\
Caipira & $321,4 \mathrm{a}$ & $425,6 \mathrm{a}$ & $561,8 \mathrm{a}$ & $642,7 \mathrm{a}$ \\
Maçã & $290,9 \mathrm{~b}$ & $415,5 \mathrm{a}$ & - & - \\
Ambrosia & $277,7 \mathrm{~b}$ & $422,6 \mathrm{a}$ & $436,3 \mathrm{c}$ & $558,2 \mathrm{~b}$ \\
Bucaneiro & $291,7 \mathrm{~b}$ & $397,8 \mathrm{a}$ & $566,3 \mathrm{a}$ & $660,7 \mathrm{a}$ \\
\hline CV (\%) & 10,40 & 9,41 & 15,79 & 11,87 \\
\hline
\end{tabular}

$\overline{\text { Médias seguidas pela mesma letra na coluna pertencem ao mesmo grupo, }}$ pelo teste de Scott-Knot, a $5 \%$ de probabilidade.

\section{CONCLUSÕES}

1-Os híbridos PV42-53, PV42-68, PV42-85, FHIA-18 e FHIA-02 apresentam bom desempenho com relação à produção e potencial para serem recomendados na região do Baixo São Francisco.

2-Os híbridos Ambrosia e Bucaneiro podem ser uma alternativa às bananas do subgrupo Cavendish por apresentarem cachos grandes e sabor semelhante.

3-O híbrido YB42-07 pode ser uma opção para áreas de cultivo de banana 'Maçã' no Baixo São Francisco, tendo potencial para ser lançado como cultivar.

\section{AGRADECIMENTOS}

À Empresa Brasileira de Pesquisa AgropecuáriaEMBRAPA, pelo apoio financeiro; ao pesquisador Hélio Wilson Lemos de Carvalho e assistentes de pesquisa José Ailton dos Santos, Inácio Roque de Andrade Júnior, José Raimundo Fonseca Freitas, Robson Silva de Oliveira e Damião Remigio de Souza, pelo apoio na execução do trabalho.

\section{REFERÊNCIAS}

ALVES, E. J.; OLIVEIRA, M. de A. Práticas culturais. In: ALVES, E. J. (Org.). A cultura da banana: aspectos técnicos, socioeconômicos e agroindustriais. 2. ed. Brasília: Embrapa-SPI/ Cruz das Almas: Embrapa-CNPMF, 1999. p. 335-352. 
DANTAS, J. L. L.; SHEPHERD, K.; SILVA, S. de O.; SOUZA, A. da S.; ALVES, E. J.; CORDEIRO, Z. J. M.; SOARES FILHO, W. dos S. Citogenética e melhoramento genético. In: ALVES, E. J. (Org.). A cultura da banana: aspectos técnicos, socioeconômicos e agroindustriais, 2. ed. Brasília: Embrapa-SPI/Cruz das Almas: Embrapa-CNPMF, 1999. p. 107-150.

DONATO, S. L. R.; SILVA, S. de O.; PASSOS, A. R.; LIMANETO, F. P.; LIMA, M. B. de. Avaliação de variedades e híbridos de bananeira sob irrigação. Revista Brasileira de Fruticultura, Jaboticabal, v. 25, n. 2, p. 348-351, 2003.

FAO 2008. Food and Agricultural Organization. Disponível em $<$ http://apps.fao.org/page/collections $>$. Acesso em: 01 jan. 2007.

FLORES, J. C. O. Avaliação de cultivares e híbridos de bananeira (Musa spp.) em quatro ciclos de produção em Cruz das Almas BA. 2000. 109 f. Dissertação (Mestrado) -Universidade Federal da Bahia, Cruz das Almas, 2000.

IBGE 2008. Disponível em <http://www.sidra.ibge.gov.br/bda/ tabela/protabl.asp>. Acesso em: 25 jan. 2008.

LIMA, M. B.; SILVA, S. de O.; JESUS, O. N. de; OLIVEIRA, W. S. J. de; AZEVEDO, R. L. de. Avaliação de cultivares e híbridos de bananeira no Recôncavo Baiano. Ciência e Agrotecnologia, Lavras, v. 29, n. 3, p. 515-520, 2005.

PEREIRA, L. V.; SILVA, S. de o.; ALVES, E. J.; SILVA, C. R. de R. Avaliação de cultivares e híbridos de bananeira em Lavras-MG. Ciência e Agrotecnologia, Lavras, v. 27, n. 1, p. 17-25, 2003.

RODRIGUES, M. G. V.; SOUTO, R. F.; SILVA, S. de O. Avaliação de genótipos de bananeira sob irrigação. Revista Brasileira de Fruticultura, Jaboticabal, v. 28, n. 3, p. 444-448, 2006.

SCARPARE FILHO, J. A.; KLUGE, R. A. Produção de bananeira 'Nanicão' em diferentes densidades de plantas e sistemas de espaçamento. Pesquisa Agropecuária Brasileira, Brasília, v. 36, n. 1, p. 105-113, 2001.
SILVA, E. A. da; BOLIANI, A. C.; CORRÊA, L. de S. Avaliação de cultivares de bananeira (musa sp) na região de Selvíria-MS. Revista Brasileira de Fruticultura, Jaboticabal, v. 28, n. 1, p. 101-103, 2006.

SILVA, S. de O.; ALVES, E. J.; SHEPHERD, K.; DANTAS, Z. L. L. Cultivares. In: ALVES, E. J. (Org.). A cultura da banana: aspectos técnicos, socioeconômicos e agroindustriais. 2. ed. Brasília: Embrapa-SPI/Cruz das Almas: Embrapa-CNPMF, 1999. p. 85105.

SILVA, S. de O.; FLORES, J. C. de O.; LIMANETO, F. P. Avaliação de cultivares e híbridos de bananeira em quatro ciclos de produção. Pesquisa Agropecuária Brasileira, Brasília, v. 37, n. 11,p. 1567-1574, 2002.

SILVA, S. de O.; PASSOS, A. R.; DONATO, S. L. R.; SALOMÃO, L. C. C.; PEREIRA, L. V.; RODRIGUES, M. G. V.; LIMANETO, F. P.; LIMA. M. B. Avaliação de genótipos de bananeira em diferentes ambientes. Ciência e Agrotecnologia, Lavras, v. 27, n. 4, p. 737-748, 2003

SILVA JUNIOR, J. F. da; MOURA, R. J. M. de; SILVA, S. de O.; GOUVEIA, J.; SANTOS, V. F. dos; LOPES JUNIOR, A. R. Evaluación de cultivares e híbridos de banano y plátano en el trópico húmedo del estado de Pernambuco, Brasil (1er. Ciclo). In: REUNIÓN DE LAASSOCIACIÓN PARA LA COOPERACIÓN EN INVESTIGACIONES DE BANANO EN EL CARIBE Y EN AMÉRICA LATINA, 15., 2002, Cartagena de Indias, Colómbia. Memorias ... Cartagena de Indias: ACORBAT, 2002. p. 441-445.

SIMÃO, S. Tratado de fruticultura. Piracicaba: FEALQ, 1998. $760 \mathrm{p}$.

VIEIRA NETO, R. D. Recomendação de variedades de bananeira para o baixo São Francisco. Aracaju: Centro de Pesquisa Agropecuária dos Tabuleiros Costeiros, 2001. 4p. (Comunicado Técnico, 44). 